\title{
Data Abstraction for CSP-OZ ${ }^{\star}$
}

\author{
Heike Wehrheim \\ Universität Oldenburg \\ Fachbereich Informatik \\ Postfach 2503, D-26111 Oldenburg, Germany \\ wehrheim@informatik.uni-oldenburg.de
}

\begin{abstract}
CSP-OZ is an integrated formal method which combines the state-oriented method Object- $Z$ with the process algebra CSP, thereby allowing a description of static as well as dynamic aspects of a system. Checking correctness of CSP-OZ specifications can be done via a translation into (FDR-)CSP, on which automatic verification can be performed with the tool FDR if the resulting CSP process is not too large to be processed. This paper investigates how data abstraction techniques can be used to bring a translated specification within range of automatic verification.
\end{abstract}

\section{Introduction}

Recently, there is an emerging interest in specification techniques that combine specification methods for different views on a system. In particular, methods integrating static aspects (data) and dynamic aspects (behaviour) are investigated (see for example [17, 10, 22, 20]). CSP-OZ [7] is a formal method that combines a method for the description of static aspects of systems, Object-Z [6] (an object-oriented extension of Z 21 24]), with a method for the specification of dynamic behaviour, the process algebra CSP [11, 18. The general idea behind this integration is to augment the state-oriented Object-Z specification with the specification of behaviour in the style of CSP while maintaining a clear separation of the issues of data and behaviour. The combination has a uniform formal semantics in the style of CSP failures and divergences.

In this paper, we will be concerned with verifying CSP-OZ specifications. The first step in the verification of a given CSP-OZ specification will be a translation into CSP1, to be precise, into the CSP dialect of the model checker FDR (Failure-Divergence-Refinement [9]). This technique has been proposed in [8], extending previous ideas of [16, 19] to use FDR to check properties of (CSP-)Z specifications. FDR-CSP is a combination of CSP with a functional language in the style of Haskell and Miranda. The functional language can be used to encode the $\mathrm{Z}$ data descriptions. Due to the possibly large data domain specified in the

\footnotetext{
* This work was partially funded by the Leibniz Programme of the German Research Council (DFG) under grant Ol 98/1-1.

${ }^{1}$ The translation is possible for a large subset of CSP-OZ, but cannot treat all Z features.
} 
Object-Z part of a CSP-OZ specification, the resulting CSP process may however be too complex to be processed by FDR. This paper investigates the usefulness of data abstraction techniques to reduce the complexity of property checking on CSP-OZ specifications. The general idea is to apply the framework of abstract interpretation [4] in the specific setting of CSP-OZ. The use of abstraction techniques for verification has already been investigated for temporal-logic model checking [5, 1, 14] and is based on abstracting transition systems (the models for various specification formalisms) while preserving properties formulated in temporal logic. Techniques for directly constructing abstract transition systems from specifications are given to avoid the construction of the (possibly large) concrete transition system at all. These techniques most often rely on choosing some abstract data domain and abstractly interpreting the operators in the program on this domain. Another application of abstract interpretation in verification is the construction of models from real software written in a programming language 3].

The work most closest to us is [2] which gives abstract interpretations for value-passing CCS where the correctness checks are based on the notion of testing. However, their abstraction results are formulated within the framework of Galois connections on transition systems, the semantic models of CCS. For a designer having written a specification in CCS, it is hard to understand the practical meaning of the result, it is formulated completely different than his specification. Furthermore, the obtained abstraction result cannot be the basis for further process algebraic manipulation. In the approach we present here, specifications, correctness checks and abstraction results are formulated in the process algebra theory of CSP. Thus we have a uniform formalism for specification and verification. A designer using the abstraction technique does not have to understand the "lower" semantic level of transition systems. Since the abstraction results are formulated within CSP theory, they can for instance be further combined (compositionality) or projected down to the concrete level. Furthermore, due to the clear separation of data and behaviour in a CSP-OZ specification, data abstraction only requires local changes of the CSP-OZ specification (or directly of the CSP process obtained by translation): changing the $\mathrm{Z}$ part by replacing concrete data domains/operations by abstract ones. We neither change the structure of the process, nor the interpretation of CSP operators. This enables us to use FDR without any additional modifications to the program or the model checking algorithm.

The paper is structured as follows: we start with a brief introduction of CSP$\mathrm{OZ}$ and the translation into CSP. The translation maintains the separation into data and behaviour. Hence the resulting CSP processes have a quite specific structure, which we call CSP-OZ form. Section 3 discusses data abstraction for CSP processes in CSP-OZ form and Section 4 gives a first example. The next section presents compositionality results for data abstraction and illustrates the applicability of the results by means of the verification of a router. The conclusion discusses further related work, especially that of Roscoe, whose book 
[18] contains a great variety of techniques to overcome the problem of state space complexity, and we discuss the issue of data independence.

\section{Prerequisites}

We start with a brief description of CSP-OZ and the specific structure of the translated specifications. The data abstraction techniques we suggest are tailored towards this specific structure. The translation will be explained through an example, details can be found in 8]. Afterwards we briefly describe the semantics underlying the process algebra CSP. A summary of CSP operators can be found in the appendix, a more detailed introduction in [11].

\section{$2.1 \quad$ CSP-OZ}

CSP-OZ is an integrated formal method that allows for a description of static aspects (by means of Object-Z) as well as dynamic aspects (by means of the process algebra CSP). A CSP-OZ specification describes a system as a collection of interacting objects, each of which has a prescribed structure and behaviour. Communication takes place via channels in the style of CSP. In general, a CSP$\mathrm{OZ}$ specification consists of a number of paragraphs, introducing classes, global variables, functions and types. CSP-OZ classes can be combined using the CSP operators parallel composition, external and internal choice and hiding; thus overriding the corresponding operators in Object-Z. In this paper, we will mainly be concerned with CSP-OZ classes, to be precise, with CSP processes which are translations of CSP-OZ classes. The specific structure of these programs can best be understood with the original CSP-OZ class structure in mind, which is as follows:

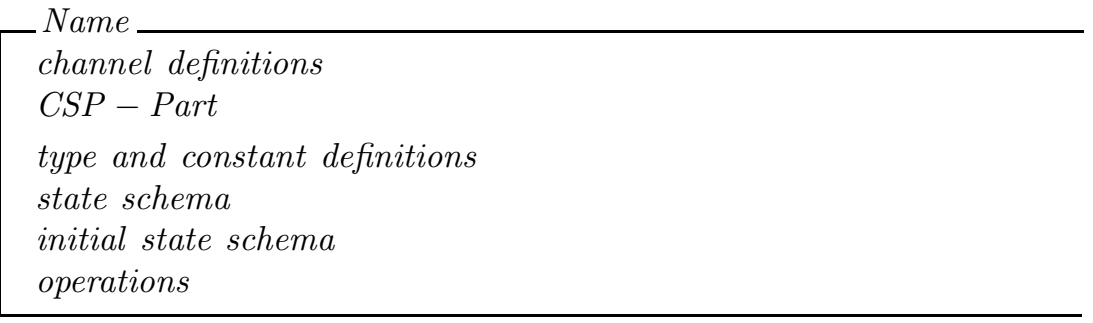

We explain the different parts of a CSP-OZ class through a simple example, the specification of a pool. The pool simply stores elements out of some element set Elem,

$$
\text { Elem }==0 . .10
$$

and allows to input and output elements. Every input is signalled to the environment. 


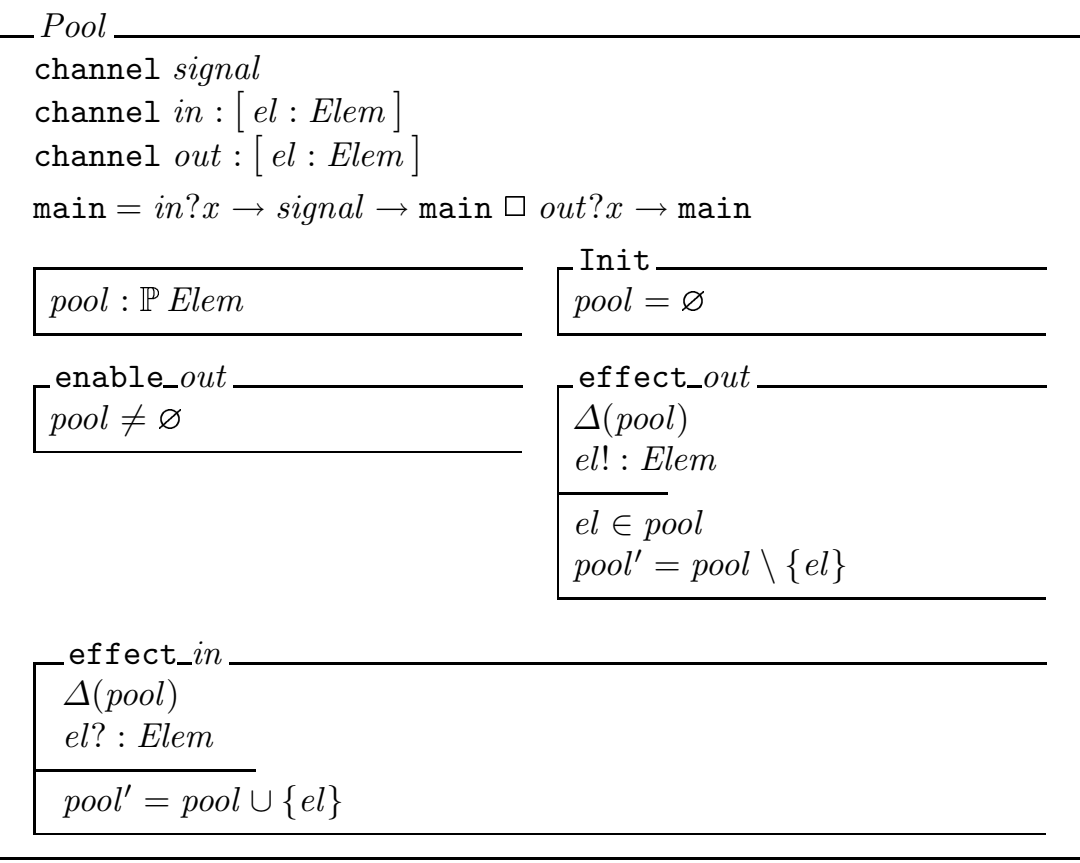

The first part defines the syntactic interface of the class, i.e. the channels which can be used for communication with the environment. The second part contains all CSP-declarations, defining the dynamic behaviour of the class; for our pool: it may either input some new elements after which this has to be signalled to the environment or $(\square)$ output some elements. No reference to data in any kind occurs in the CSP part; all communications are of the form ch? $\mathrm{x}$ (a communication of the form $\mathrm{ch}$ ! e would refer to some concrete data value). Thus also the output of our pool has the form out?x.

The remaining part of the class definition contains Object-Z-declarations. The main part is the declaration of the state schema (here the state variable pool), the initial state schema (its initial value), and operation schemas. The definition of an operation has to be divided into an (optional) enabling and an effect schema. The enabling schema (e.g. enable_out) is a predicate over the state variables of the class, and describes the states in which the operation can be executed (e.g. outputs are only possible when the pool is non-empty). Enable schemas are thus used to describe data-dependent behaviour. The effect schema (a predicate over the state variables and input parameters) describes the effect of an operation on the state space and the values of possible output parameters (the primed variables are used to describe the value of a variable in the next state, the $\Delta$-list lists all variables which are changed by the operation). 


\subsection{Structure of Translated CSP-OZ Specifications}

For the verification, every class of a CSP-OZ specification is translated into the definition of a CSP process. When using such a definition, we will also refer to this as the CSP process of an object, a particular instance of a class.

The translation preserves the clear separation between data and behaviour. The CSP part of the CSP-OZ specification remains unchanged while the Z part has to be translated into CSP. Intuitively, the behaviour of the $\mathrm{Z}$ part is as follows: in every state (specific values of the state variables) one of the enabled operations may be taken and the resulting state is determined by the effect of the operation. Thus the CSP translation of the Z part is an external choice over all class operations with their enabling conditions as guards. The effect schemas, which are predicates in the CSP-OZ specification, are translated into functions from state space and input parameters to sets of possible new states and values of output parameters. The resulting new state and the values for output parameters are nondeterministically chosen out of this effect set of an operation.

Assume that we are given a CSP-OZ class specification $C L S$ with state variables $v_{1}, \ldots, v_{n}$ in the state schema, initial values $\operatorname{ini}\left(v_{i}\right)(1 \leq i \leq n)$ and set of operations $O p s$, and that we may derive a function In assigning to every channel $c h \in$ Ops its possible values of input variables (derived from the type of the channel), then the translated specification has the following form (using partly FDR-CSP and CSP syntax to improve readability; for a description of FDR-CSP see appendix):

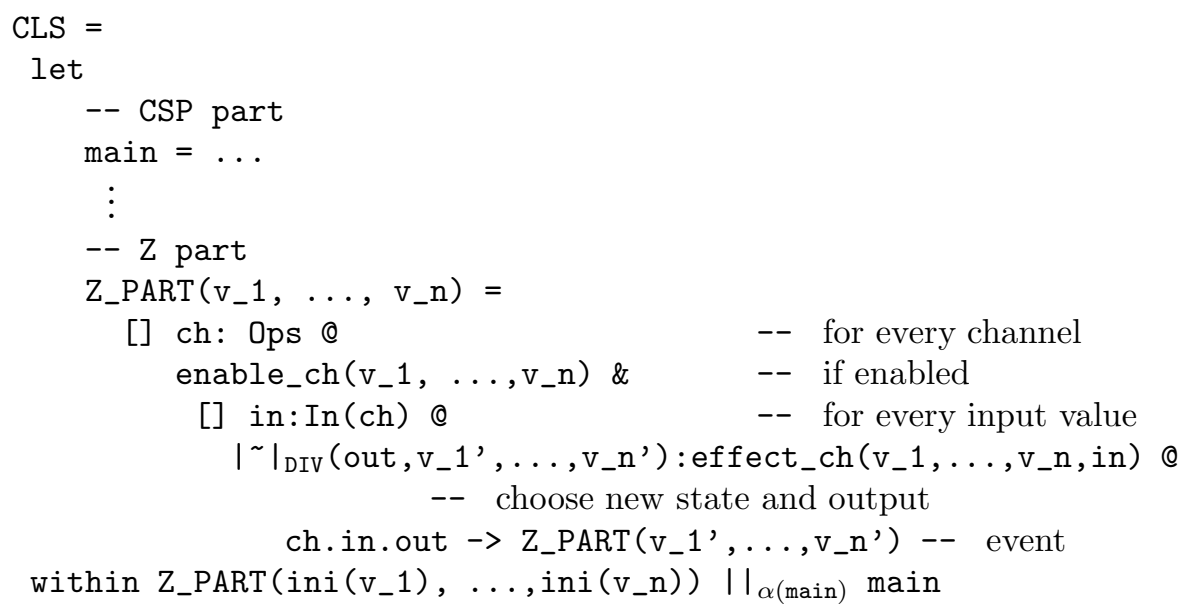

The abbreviation $\alpha$ (main) stands for the alphabet of the process main (the set of channel names occurring in it) and $\left.I^{\sim}\right|_{\text {DIV }}$ is a special internal choice operator capturing the case that the index set of the iterated $I^{\sim} \mid$ is empty, in this case the process diverges. The intuition behind the use of this operator is the following: if an operation is enabled but its effect is not defined for the current state (yielding an empty effect set), the process behaves as completely undetermined, it diverges.

The structure of the translated class can be further simplified when there are no input or no output parameters of a channel, or when the effect of an 
operation is deterministic, i.e. the effect set contains a single value. None of our examples will use the general structure, most often the $\mathrm{Z}$ part is simply of the form guard1 \& ch_1 $\rightarrow \mathrm{Z}(\ldots)$ [] guard2 \& ch_2 $\rightarrow \mathrm{Z}(\ldots) \ldots$

The translation of the class Pool given above is:

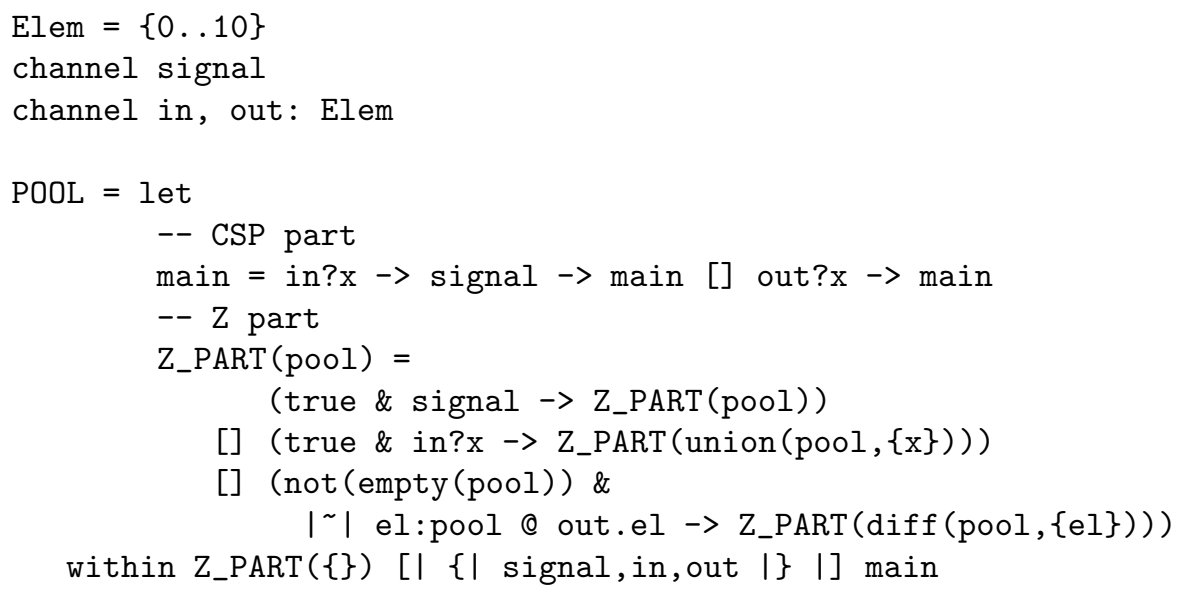

\subsection{CSP Semantics}

The standard semantic model of CSP is the failure-divergence model. The same model has been used in [7] to give a semantics to CSP-OZ, by defining a failuredivergence semantics for the $\mathrm{Z}$ part. Thus a uniform semantics is achieved. An alternative weaker semantic model which is also supported by FDR is the trace model. We will use both models here. The trace model is sufficient for studying safety properties, while for liveness properties (e.g. deadlock or livelock freedom) a more discriminating semantics has to be chosen. Traces record the possible runs of a process, failures additionally give sets of events that are refused after some run, and divergences describe the set of traces after which the process may diverge, i.e. perform an infinite number of internal events. Thus, given a set of events $\Sigma$ (typically of the form $c h . v$, where $c h$ is a channel name and $v$ a value), we have

$$
\begin{aligned}
\operatorname{traces}(P) & \subseteq \mathbf{2}^{\Sigma^{*}} \\
\text { failures }(P) & \subseteq \mathbf{2}^{\Sigma^{*} \times \mathbf{2}^{\Sigma}} \\
\text { divergences }(P) & \subseteq \mathbf{2}^{\Sigma^{*}}
\end{aligned}
$$

These semantic models are used to compare processes and check properties on them. The most important comparison concept in CSP theory is refinement:

Definition 1. A CSP process $P_{1}$ is a failure-divergence refinement of a process $P_{2}$ (denoted $P_{2} \sqsubseteq \mathcal{F} P_{1}$ ) if

$$
\text { failures }\left(P_{2}\right) \supseteq \operatorname{failures}\left(P_{1}\right) \text { and divergences }\left(P_{2}\right) \supseteq \operatorname{divergences}\left(P_{1}\right) \text {. }
$$

They are failure-divergence equivalent $\left(P_{1}={ }_{\mathcal{F}} P_{2}\right)$ if $P_{1} \sqsubseteq \mathcal{F} P_{2}$ and $P_{2} \sqsubseteq \mathcal{F} P_{1}$. 
If $P_{1}$ is a refinement of $P_{2}$, it can be seen as an implementation of $P_{2}$ since it is more deterministic than $P_{2}$. A weaker refinement notion is obtained when only the traces are used for comparison:

Definition 2. A CSP process $P_{1}$ is a trace refinement of a process $P_{2}$ (denoted $\left.P_{2} \sqsubseteq \mathcal{T} P_{1}\right)$ if traces $\left(P_{2}\right) \supseteq \operatorname{traces}\left(P_{1}\right)$.

Both refinement notions are compositional (or monotone): given two processes $P_{1}, P_{2}$ and a CSP context $C[\cdot]$, then $P_{1} \sqsubseteq P_{2} \Rightarrow C\left[P_{1}\right] \sqsubseteq C\left[P_{2}\right]$, where $\sqsubseteq \epsilon$ $\{\sqsubseteq \mathcal{T}, \sqsubseteq \mathcal{F}\}$.

There are two possibilities for deriving the semantics of a CSP process: by a denotational semantics which compositionally computes traces, failures and divergences, or via a structured operational semantics which constructs a transition system for a process, from which traces, failures and divergences are computed. Both semantics are consistent: they compute the same traces, failures and divergences of a process. We refer to [18]) for details. A summary of CSP operators in FDR syntax can be found in the appendix. The two operators which will be used in our results are

- parallel composition with sychronisation on some set of events $A$ : $\|_{A}$, and

- renaming: $[R]$, which renames all events according to the renaming relation $R$.

We use general renaming relations, not just injective functions. As an example for a CSP process with renaming: Let $P=a \rightarrow S K I P$ and $R=\{(a, b),(a, c)\}$, then $P[R]$ is equal to $b \rightarrow S K I P \square c \rightarrow S K I P$.

\section{Data Abstraction}

Since CSP-OZ specifications may contain rather large amounts of data, the state space of the resulting CSP process can often be too large to be processed by FDR. Several techniques have already been proposed to overcome this problem; especially in the book of Roscoe [18] several methods can be found together with various application examples.

The technique we propose here is based on abstract interpretation of programs 4] and can be seen as complementing the other techniques. Abstract interpretation is a technique for program analysis which is often used in compiler design for static analysis (e.g. data-flow analysis, strictness analysis, etc.). The results of an abstract interpretation can for instance be used in type checking or optimisation. The idea of abstract interpretation is to interpret a program in an abstract domain using abstract operations. The main advantage is that the concrete program does not have to be executed while still being able to obtain information about its real execution. For verification of formal specifications, the basis of abstract interpretation is to construct an abstract model of the specification on which abstract properties can be proven which give information on the concrete model [5, 1] 14]. For this, the data domain of the concrete program 
has to be abstracted and operations of the program are abstractly interpreted on the new data domain.

In order to apply data abstraction techniques to CSP-OZ, we first have to make clear what the data is we want to abstract, and what the operations are we want to interpret abstractly. Since the goal is to use FDR for automatic verification of properties on the abstracted systems, we certainly cannot change the semantics of CSP operators in any way. What may be interpreted abstractly are the enable and effect operations coming from the $\mathrm{Z}$ part of the CSP-OZ specification. The relevant data domains to be abstracted are the domains of the state variables: $D_{1}, \ldots, D_{n}$ for variables $v_{1}, \ldots, v_{n}$, and the domains of the channels: $M_{1}, \ldots, M_{k}$ for channels $c h_{1}, \ldots, c h_{k}$. We assume that a domain of a channel $c h_{i}$ is split into a domain for input parameters $M_{i}^{i n}$ and for output parameters $M_{i}^{\text {out }}$. Then the enable and effect operations have the following signature (we will refer to this concrete semantics of enable and effect by using the semantic brackets $\llbracket \cdot \rrbracket)$ :

$$
\begin{array}{ll}
\llbracket \text { enable_ch } \_: D_{1} \times \ldots \times D_{n} & \rightarrow \mathbb{B} \\
\llbracket \text { effect_ch }{ }_{i} \rrbracket:\left(D_{1} \times \ldots \times D_{n} \times M_{i}^{\text {in }}\right) & \rightarrow \mathbf{2}^{M_{i}^{\text {out }} \times D_{1} \times \ldots \times D_{n}}
\end{array}
$$

Instead of interpreting the CSP program on these concrete data domains with the concrete meaning of enable and effect operations, we use abstract data domains and abstract interpretations of enable and effect. For this, we first choose abstract data domains $D_{i}^{A}$ and $M_{j}^{A}$ and abstraction functions:

$$
\begin{aligned}
h_{i}: D_{i} & \rightarrow D_{i}^{A} \\
g_{j}: M_{j} & \rightarrow M_{j}^{A}
\end{aligned}
$$

We define $h\left(d_{1}, \ldots d_{n}\right):=\left(h_{1}\left(d_{1}\right), \ldots, h_{n}\left(d_{n}\right)\right)$ and let $(h, g)\left(d_{1}, \ldots, d_{n}, m\right)$ stand for $\left(h_{1}\left(d_{1}\right), \ldots, h_{n}\left(d_{n}\right), g(m)\right)$. In the following, we will abbreviate $\left(d_{1}, \ldots, d_{n}\right)$ simply by $d$. For a given abstraction function $g$ of channel values, we let $G$ denote the corresponding renaming function on events: $G\left(c h_{i} \cdot w\right)=c h_{i} \cdot g(w)$.

An abstract interpretation $\llbracket \cdot \rrbracket^{A}$ of enable and effect operations operates on abstract data domains:

$$
\begin{array}{ll}
\llbracket \text { enable_ch } \rrbracket^{A}: D_{1}^{A} \times \ldots \times D_{n}^{A} & \rightarrow \mathbb{B} \\
\llbracket \text { effect_ch } \rrbracket^{A}:\left(D_{1}^{A} \times \ldots \times D_{n}^{A} \times M_{i}^{\text {in }} A\right. & \rightarrow \mathbf{2}^{M_{i}^{\text {out } A} \times D_{1}^{A} \times \ldots \times D_{n}^{A}}
\end{array}
$$

In order to use FDR as a model-checker on abstracted systems, we have to replace the concrete enable and effect predicates by abstract predicates enable $A$

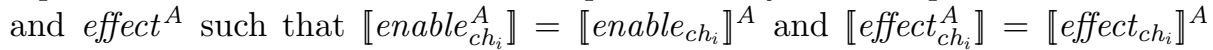
holds, i.e. the concrete semantics of the new enable and effects must equal the abstract semantics of the old ones. Furthermore channels have to be declared over the abstract domains. These changes can already be done in the CSP-OZ specification, the designer does not have to look at the CSP code at all.

So far, we are free to use whatever abstract interpretation we want. Of course, the abstracted system should somehow reflect the behaviour of the concrete system: we want to abstractly observe the events of the concrete system. To 
ensure this, we have to impose conditions on the abstract interpretations. We consider two types of abstract interpretations: safe and optimal interpretations.

Definition 3. An abstract interpretation $\llbracket \cdot \rrbracket^{\mathcal{S}}$ of enable and effect predicates is safe with respect to abstraction functions $h$ and $g$ iff

$$
\forall d \in D^{A}: \llbracket e n a b l e_{-} c h_{i} \rrbracket^{\mathcal{S}}\left(d^{A}\right) \Leftrightarrow \exists d \in D: h(d)=d^{A} \wedge \llbracket e n a b l e_{-} c h_{i} \rrbracket(d)
$$

and

$$
\begin{gathered}
\forall d \in D^{A}, m \in M^{A}: \\
\llbracket \text { effect_ch } \rrbracket_{i} \rrbracket^{\mathcal{S}}\left(d^{A}, m^{A}\right)=\bigcup_{\substack{(d, m) \in D \times M \\
h(d)=d^{A}, g_{i}(m)=m^{A}}}\left(h, g_{i}^{\text {out }}\right)\left(\llbracket \text { effect_ch } h_{i} \rrbracket(d, m)\right)
\end{gathered}
$$

A safe abstract interpretation guarantees that in a state of the abstract system a communication over some channel is possible whenever there is some corresponding concrete state in which this communication is enabled. The result of a safe abstraction is an abstract system that allows more moves than the concrete system. An abstraction which more faithfully represents the concrete system, is an optimal abstraction.

Definition 4. An abstract interpretation $\llbracket \cdot \rrbracket^{\mathcal{O}}$ of enable and effect predicates is optimal with respect to abtraction functions $h$ and $g$ iff

$$
\begin{aligned}
\forall d \in D^{A}: \llbracket \text { enable_ch }_{i} \rrbracket^{\mathcal{O}}(h(d)) & \Leftrightarrow \llbracket \text { enable_ch }{ }_{i} \rrbracket(d) \quad \text { and } \\
\forall d \in D^{A}, m \in M^{A}: \llbracket e f f e c t_{-} c h_{i} \rrbracket^{\mathcal{O}}\left(h(d), g_{i}(m)\right) & =\left(h, g_{i}^{\text {out }}\right)\left(\llbracket \text { effect_ch } h_{i} \rrbracket(d, m)\right.
\end{aligned}
$$

An optimal abstracted system exactly mimics the behaviour of the concrete system, only the precise values of communication cannot be observed anymore. Depending on the choice of abstraction functions, it may not be possible to find an optimal abstract interpretation. Optimality requires abstraction functions which guarantee that all concrete states which are abstracted into the same abstract state abstractly behave "the same". Note that every optimal abstraction is safe. Tool support for proving optimality or safety of interpretations is available in the form of theorem provers for $\mathrm{Z}$ [13, 15].

Given a specification $S$, we let $S^{\mathcal{S}}$ refer to a safe and $S^{\mathcal{O}}$ to an optimal abstraction of $S$.

What is now the relationship between the behaviour of the abstracted and the concrete system? Our goal is to express this relationship completely in terms of process algebra notions. This enables us to use all of the process algebra theory for further manipulation of the result. In the abstracted system we can, of course, only observe communications with abstract values. This is the nature of abstract interpretation, we have lost some information about the concrete system, in our case the concrete values of communication. Thus we can only compare the abstracted system with the renamed concrete system: 
Theorem 1. Let $S$ be a CSP process in CSP-OZ form, $g_{1}, h_{1}$ abstraction functions for a safe and $g_{2}, h_{2}$ for an optimal interpretation. Then the following holds:

$$
\begin{aligned}
S^{\mathcal{S}} & \sqsubseteq \mathcal{T} S\left[G_{1}\right] \quad \text { and } \\
S\left[G_{2}\right] & =\mathcal{F} S^{\mathcal{O}} .
\end{aligned}
$$

The proof can be found in the appendix. This result can be the basis for further process algebraic computations; for instance compositionality of trace refinement immediately gives us $\left(S^{\mathcal{S}}\right)\left[G^{-1}\right] \sqsubseteq \mathcal{T}(S[G])\left[G^{-1}\right]$. The latter is equal to $S[G \circ$ $\left.G^{-1}\right]$ which itself can easily be shown to be trace refined by $S: S\left[G \circ G^{-1}\right] \sqsubseteq \mathcal{T} S$. All in one, we therefore get:

$$
\left(S^{\mathcal{S}}\right)\left[G^{-1}\right] \sqsubseteq \mathcal{T} S
$$

This result refers directly to the concrete system $S$. With the help of this abstraction theorem, we are now able to proof properties of a CSP-OZ class $S$ in the following way:

- construct an abstract class specification: choose abstract domains, abstraction functions and abstract enable and effect predicates,

- show safety/optimality of abstraction (supported by some theorem prover extension for Z),

- translate the abstract specification into FDR-CSP (in future automatic),

- show property for $S^{\mathcal{S}} / S^{\mathcal{O}}$ (FDR), e.g. Prop $\sqsubseteq \mathcal{T} S^{\mathcal{S}}$,

- conclude the holding of a concretised property for $S$ (abstraction theorem), e.g. $\operatorname{Prop}\left[G^{-1}\right] \sqsubseteq \mathcal{T} S$.

Most of these steps in the verification are tool-supported or even automatic. Nevertheless, the crucial part of abstraction, finding good abstract domains and abstraction functions, is still left to the user. 1] gives some heuristics for the choice of abstraction functions.

\section{First Example: An Optimal Abstraction}

The following example gives a first impression of data abstractions for CSPOZ. It is an optimal abstraction, that reduces an equivalence check between an infinite state and a finite state specification to an equivalence check between two finite state systems. Although both systems are very simple, this already shows the potential of abstract interpretation.

Both specifications describe a simple clock with alternating tick and tock events. While in the first clock the alternation is encoded by the usage of enabling predicates (the $\mathrm{Z}$ part uses a counter),

$$
\text { Clock1 }=\text { let }
$$




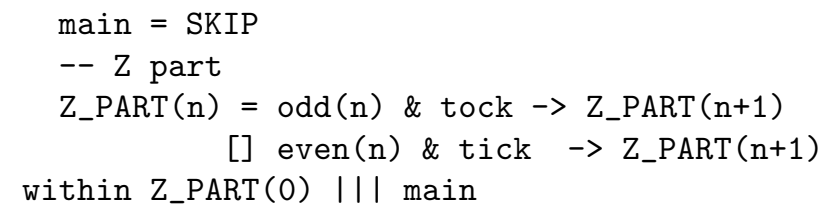

the second clock encodes alternation in the CSP part.

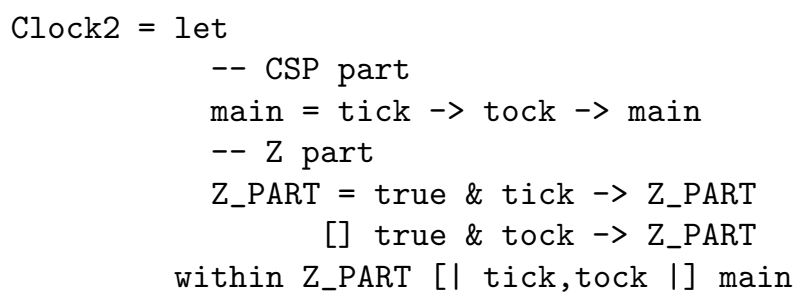

Hence an equivalence proof of both clocks by separate data and process refinement proofs is not possible. FDR can also not be used since the state space of the first clock is infinite.

We now apply the following abstraction function $h$ to the first clock

$$
h: k \mapsto \begin{cases}0 & \text { if } \operatorname{even}(k) \\ 1 & \text { if } \operatorname{odd}(k)\end{cases}
$$

and replace the enabling and effect operations by their following abstract versions: $(+1)^{A}(k):=(+1)(k) \bmod 2$, even $^{A}(k):=\operatorname{even}(k)$, odd $^{A}(k):=\operatorname{odd}(k)$. This is an optimal abstract interpretation for Clock1 with respect to $h$. Note that in this case no abstraction function for channel values is needed and thus the renaming function $G$ of Theorem $\Pi$ is empty. Thus we get the first result: Clock $1^{\mathcal{O}}=_{\mathcal{F}}$ Clock 1 . The abstracted clock is now finite state, thus we can use FDR for checking whether $\operatorname{Clock} 1^{\mathcal{O}}=_{\mathcal{F}}$ Clock2 (the answer is yes), and this implies Clock $1=_{\mathcal{F}}$ Clock 2 .

This rather simple example (without input and output parameters) also reveals close similarities between data abstraction and data refinement (which is the standard refinement notion within $\mathrm{Z}$ ). The data abstraction we have used here is in fact also a valid data refinement (from $C l o c k 1$ to $C l o c k 1^{\mathcal{O}}$ and vice versa). In general, in our setting every optimal data abstraction can also be seen as a data refinement plus a renaming.

\section{Compositionality}

In this section, we will be concerned with the issue of compositionality of data abstractions. Two aspects will be considered here:

- combining abstraction results for different objects of a system, and

- combining different abstractions of the same object.

The results we get here are formulated within the traces model, thus we only deal with safety properties. 


\subsection{Composing Objects}

In general, we will not be interested in a single object alone, but in a system which is composed out of a number of objects operating in parallel. Thus we have to extend our abstraction theorem to parallel compositions of objects. Two aspects are helpful for this extension: the first is the fact that the abstraction theorem for objects is completely formulated within process algebra theory, and the second is the compositionality (monotonicity) of both trace and failure refinement.

Consider a system $S$ composed out of two objects $S_{1}$ and $S_{2}$ operating in parallel with synchronisation on some set $A \subseteq \Sigma: S=S_{1} \|_{A} S_{2}$. So far, we are able to prove properties of $S_{1}$ and $S_{2}$ alone by for instance using some safe abstraction with respect to abstraction functions $h_{1}, g_{1}, h_{2}, g_{2}$ respectively. By our abstraction theorem, we know that $S_{1}^{\mathcal{S}} \sqsubseteq_{\mathcal{T}} S_{1}\left[G_{1}\right]$ and $S_{2}^{\mathcal{S}} \sqsubseteq_{\mathcal{T}} S_{2}\left[G_{2}\right]$. The first prerequisite for a combination of the abstracted objects is the preservation of their communication ability: the abstraction functions for the channel values have to agree on joint channels.

Definition 5. Let $g_{1}, g_{2}$ be abstraction functions for $S_{1}$ 's and $S_{2}$ 's channel values and let $A=\alpha\left(S_{1}\right) \cap \alpha\left(S_{2}\right)$ be the set of joint events, with channel names Ch in $A . g_{1}$ and $g_{2}$ agree on A iff for all $c h \in C h, v \in D_{c h}, g_{1, c h}(v)=g_{2, c h}(v)$.

When the abstraction functions of the components agree on the joint events of the components, we can look at the abstracted system as $S^{\mathcal{S}}=S_{1}^{\mathcal{S}} \|_{G_{1}(A)} S_{2}^{\mathcal{S}}$. Compositionality of trace refinement already gives us the following result:

$$
S_{1}^{\mathcal{S}}\left\|_{G_{1}(A)} S_{2}^{\mathcal{S}} \sqsubseteq \mathcal{T} S_{1}\left[G_{1}\right]\right\|_{G_{1}(A)} S_{2}\left[G_{2}\right]
$$

However, the left hand side is not the system we are actually interested in, namely $S_{1} \|_{A} S_{2}$. The next proposition helps us towards this goal.

Proposition 1. Let $f: \Sigma \rightarrow \Sigma$ be a renaming function and $A \subseteq \Sigma$ a set of events such that $a \in A \Leftrightarrow f(a) \in f(A)$ holds; let $P_{1}, P_{2}$ be CSP processes. Then

$$
P_{1}[f] \|_{f(A)} P_{2}[f] \sqsubseteq \mathcal{T}\left(P_{1} \|_{A} P_{2}\right)[f]
$$

All renaming functions $G$ generated by some abstraction function $g$ have the above stated property, thus we immediately get:

$$
S_{1}\left[G_{1}\right] \|_{G_{1}(A)} S_{2}\left[G_{2}\right] \sqsubseteq_{\mathcal{T}}\left(S_{1} \|_{A} S_{2}\right)\left[G_{1} \cup G_{2}\right]
$$

Combining these two parts, we get the following compositionality result:

Corollary 1. Let $S_{1}, S_{2}$ be CSP processes in CSP-OZ form, $g_{1}, g_{2}$ abstraction functions which agree on joint events of $S_{1}$ and $S_{2}$. Then

$$
S_{1}^{\mathcal{S}} \|_{G_{1}(A)} S_{2}^{\mathcal{S}} \sqsubseteq \mathcal{T}\left(S_{1} \|_{A} S_{2}\right)\left[G_{1} \cup G_{2}\right] .
$$

Thus we have extended our abstraction theorem for safe abstractions to parallel compositions of objects. Unfortunately, this result cannot be extended to optimal abstractions in the sense, that we may replace trace refinement by failures equivalence. Proposition 1 does not even hold for trace equivalence since the concrete components may fail to synchronise while their abstractions communicate. 


\subsection{Combining Abstractions}

Besides compositions of objects, we are also interested in composing different abstraction results of the same object. The idea is to use different abstractions to prove different properties of an object and afterwards combine these abstractions to show that also their combination holds.

Consider an object $S$ and two different safe abstractions wrt. $h_{1}, g_{1}$ and wrt. $h_{2}, g_{2}$. We prove two properties of the object via abstractions, using FDR to show Prop $1 \sqsubseteq_{\mathcal{T}} S^{\mathcal{S}_{1}}$ and Prop $_{2} \sqsubseteq \mathcal{T} S^{\mathcal{S}_{2}}$. With the abstraction theorem and monotonicity of trace refinement we get $\operatorname{Prop}_{1}\left[G_{1}^{-1}\right] \sqsubseteq_{\mathcal{T}} S$ and $\operatorname{Prop}_{2}\left[G_{2}^{-1}\right] \sqsubseteq_{\mathcal{T}} S$ which can again be combined to give

$$
\operatorname{Prop}_{1}\left[G_{1}^{-1}\right]\left\|_{\alpha(S)} \operatorname{Prop}_{2}\left[G_{2}^{-1}\right] \sqsubseteq \mathcal{T} S\right\|_{\alpha(S)} S
$$

Furthermore, $S \|_{\alpha(S)} S={ }_{T} S$. Combining these two parts, we obtain as a corollary:

Corollary 2. Let $S$ be a CSP process in CSP-OZ form, $g_{1}, g_{2}$ abstraction functions for its channels, Prop 1 , Prop 2 arbitrary CSP processes. Then

$$
\operatorname{Prop}_{1}\left[G_{1}^{-1}\right] \|_{\alpha(S)} \operatorname{Prop}_{2}\left[G_{2}^{-1}\right] \sqsubseteq_{\mathcal{T}} S .
$$

Afterwards we can use process algebra theory to compute the overall property Prop $=\operatorname{Prop}_{1}\left[G_{1}^{-1}\right] \|_{\alpha(S)} \operatorname{Prop}_{2}\left[G_{2}^{-1}\right]$. When the abstractions have been carefully chosen (and when they fit together well), Prop might indeed be the property of interest. The following example demonstrates the use of both kinds of compositionality results.

\subsection{Illustrating Example}

The example is a 1 -to- $2^{n}$ router with $n$ stages (Figure 1 shows a router with two stages). Stage $i$ contains $2^{i}$ switches which one input (in) and two outputs (out 0 , out 1 ) each.

The messages which are send through the router consist of an address (a binary number of length $n$ ) and a data part. The address is used for routing: a switch at stage $i$ looks at the $i$-th bit of the address; when it is 0 it sends the message to the upper chan-

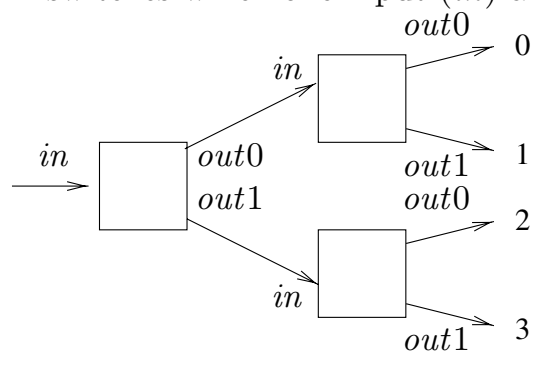

Fig. 1. A 1-to- $2^{n}$ router nel (out0), otherwise to the lower channel (out1). Receipt of messages is acknowledged (ack) and the switches delay the next input until an acknowledge has been received (rack). Thus the router contains at most one message. The following CSP specification in CSP-OZ form describes a router with 2 stages. 


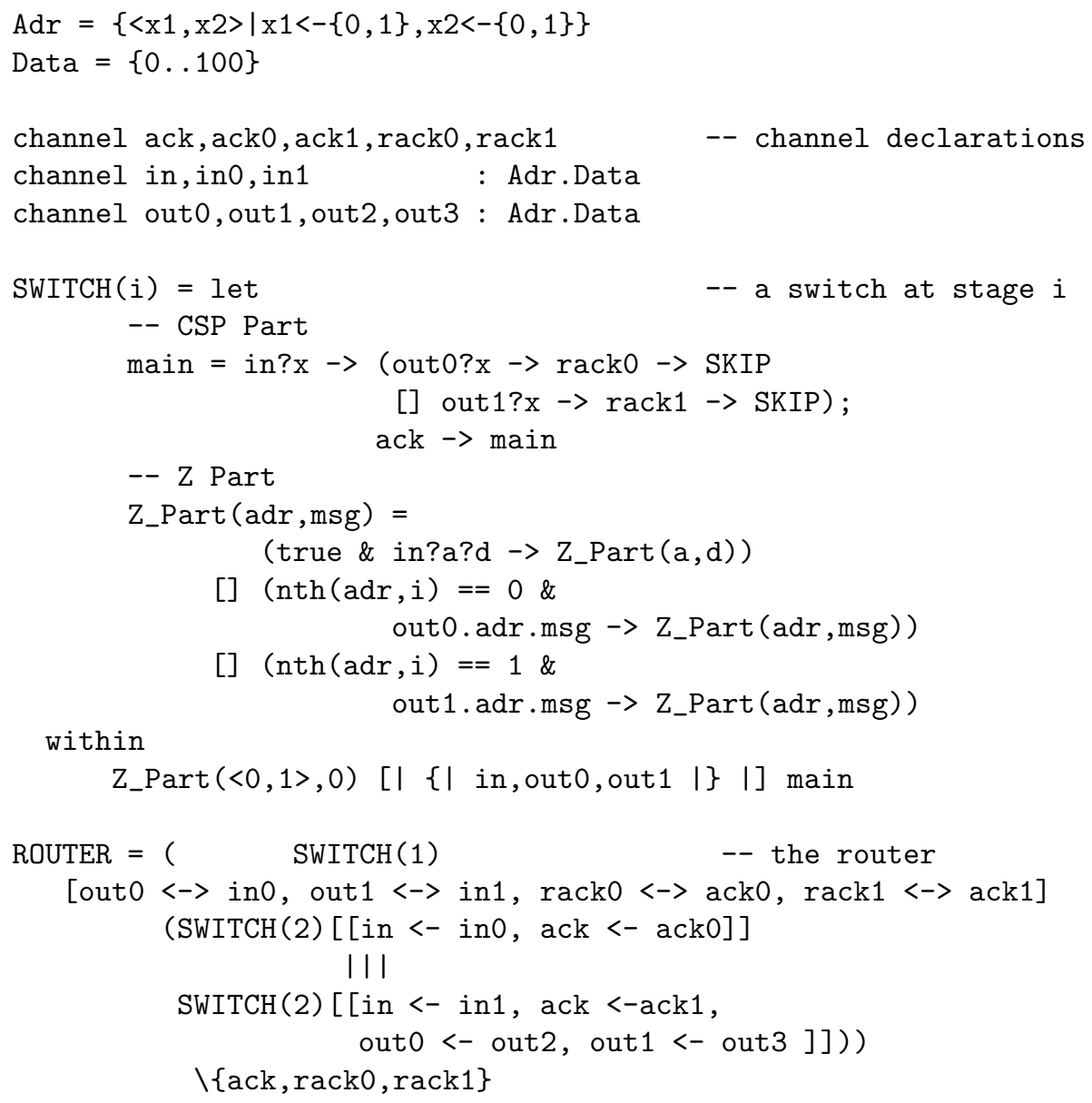

The routing within a switch is encoded in the enabling conditions of output communications out 0 and out 1 . The ordering of events (first input, then output, receive acknowledge and acknowledge itself) is encoded in the CSP process main. The switches are combined into router by renaming the corresponding ports appropriately and synchronising on them 2 . The output ports of the second stage are renamed into out 0 , out 1 , out 2 and out 3 .

The property to be verified is a safety property: every message input to the router with address $a$ and data $d$ is, if it is delivered at all, correctly delivered at output port num $(a)$ (the number with binary encoding $a$ ). Formulated within CSP: we would like to show that BUF $\subseteq_{\mathcal{T}}$ ROUTER, where BUF is defined as

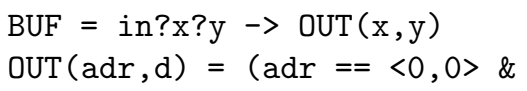

${ }^{2}$ The composition operators used in ROUTER also involve hiding (linked parallel), for which we have not established a compositionality result. However, hiding of channels commutes with renaming of channel values, i.e. $(S \backslash a)[G]=S[G] \backslash a$. 


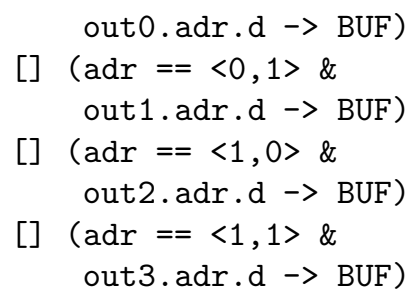

However, it may not be possible to use FDR to prove this property since the router may be too large to be processed with FDR when the domain of Data is large.

Two abstractions are possible: abstracting from the data, i.e. mapping all different data values onto one single value (functions $h_{1}, g_{1}$ ), or abstracting from the address, mapping all addresses onto a single one (functions $h_{2}, g_{2}$ ). The first abstraction allows for an optimal abstract interpretation of enable and effects (the enabling conditions are independent of the data), the latter only for a safe abstraction. For the optimal abstraction the only change in the CSP code is a change of the definition of Data, for the safe abstraction we have to change Adr (to a single-value type $<0>$ ) and the enabling conditions for out 0 and out 1 which are now both true. We now separately prove two properties:

- if a message with address $a$ is received, the next message is delivered at port $\operatorname{num}(a)$, and

- if a message with data $d$ is received, the next delivered message contains data $d$.

The formulation of these two properties in CSP is:

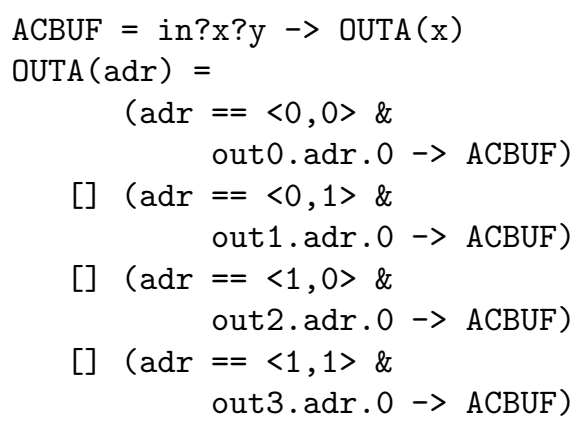

$\operatorname{DCBUF}=$ in? $\mathrm{x} ? \mathrm{y} \rightarrow \operatorname{OUTD}(\mathrm{y})$

$\operatorname{OUTD}(d)=$

out $0 .<0\rangle . d \rightarrow$ DCBUF

[] out $1 .<0\rangle . d \rightarrow$ DCBUF

[] out $2 .\langle 0\rangle . d \rightarrow$ DCBUF

[] out3. $\langle 0\rangle . d \rightarrow$ DCBUF

and we ask FDR whether the following two assertions are valid (a check for trace refinement):

$$
\begin{aligned}
& \text { assert ACBUF }[\mathrm{T}=\text { ROUTERA1 } \\
& \text { assert DCBUF }[\mathrm{T}=\text { ROUTERA2 }
\end{aligned}
$$

The answer is yes. This so far proves that $\operatorname{ACBUF}\left[G_{1}^{-1}\right] \sqsubseteq \mathcal{T}$ ROUTER and $\operatorname{DCBUF}\left[G_{2}^{-1}\right] \sqsubseteq \mathcal{T}$ ROUTER. Combining these two abstractions we get:

$$
\operatorname{ACBUF}\left[G_{1}^{-1}\right] \|_{\alpha(\text { ROUTER })} \operatorname{DCBUF}\left[G_{2}^{-1}\right] \sqsubseteq_{\mathcal{T}} \text { ROUTER }
$$


It is easy to prove within process algebra theory (by giving a divergence respecting bisimulation relation) that $\operatorname{ACBUF}\left[G_{1}^{-1}\right] \|_{\alpha \text { (ROUTER) }} \operatorname{DCBUF}\left[G_{2}^{-1}\right]$ is failures equivalent to BUF. Thus we have proven the desired property: BUF $\sqsubseteq_{\mathcal{T}}$ ROUTER. For the here chosen domain for Data, the refinement check is still possible for FDR; nevertheless the abstraction reduces a check of 16975474 states in about 170 minutes (on a SUN Ultra 2C with two processors at $300 \mathrm{MHZ}$ each and 896 $\mathrm{MB})$ to one of 1152511 states in 5 minutes.

\section{Conclusion}

In this paper we have proposed a data abstraction technique for CSP-OZ, which was based on the idea of abstract interpretation of specifications. We have shown how properties of concrete specifications are preserved by abstraction by relating the abstract to the renamed concrete specification. We studied compositionality of class abstractions and showed how abstraction results can be combined to give more information about the concrete system. The advantage of our method is that it is both simple to carry out (abstraction requires only small local changes in the CSP-OZ specification) and the results are easy to interpret. Specification, verification and abstraction is formulated within the CSP setting.

So far, the object-oriented nature of the specification language has played no role in the verification. We intend to further investigate how the object-oriented structuring of specifications can be exploited to facilitate verification.

Related work. Besides the work already discussed in the introduction, we want to further comment on some related work, especially on the work of Roscoe, both because it deals with CSP and because of its great variety of techniques. The book [18] presents several methods that can be used to analyse CSP programs which are per se too large to be processed with FDR. These techniques include

- abstraction techniques based on hiding,

- local deadlock analysis and

- data independent property checks.

Especially the last point is of interest for data abstraction: data independence (first studied by Wolper [23]) is concerned with the behaviour of programs independent of some of their parameters. For instance, one might be interested in knowing whether a buffer specification works like a buffer independently of the stored elements. Roscoe 18 reports on some work (together with Lasic) that allows to compute thresholds on the size of data domains used for particular parameters, such that it is sufficient to check a property on a given program with parameters instantiated with a domain of this size and conclude correctness for all instantiations with larger domains. This could very much facilitate correctness checks for CSP programs, since it is to be expected that usually a data independent program with a small data domain already exhibits all the "relevant" behaviour. The router example could profit from this technique; it is possibly sufficient to check the trace refinement on a domain of Data of size 2 . 
The clock example is, however, not amenable to such techniques since it is not data independent: it contains tests on data values (even, odd).

Data independence has also been an issue in other work on verification; a very interesting approach in a process algebraic setting is [12. Jonsson and Parrow study programs which are completely data independent (no modification on data, no testing of values) and show that bisimulation is decidable on this class of systems, even when the data domain is infinite. Their idea could possibily also be used for refinement, but is limited to this restricted class.

Acknowledgements. Many thanks to E.-R.Olderog, C.Fischer, H.Dierks and J.Bredereke for discussions on the paper.

\section{References}

[1] E.M. Clarke, O. Grumberg, and D.E. Long. Model checking and abstraction. In 19th ACM POPL, 1992.

[2] R. Cleaveland and J. Riely. Testing-based abstractions for value-passing systems. In B. Jonsson and J. Parrow, editors, CONCUR'94, volume 836 of Lecture Notes in Computer Science, pages 417-432, 1994.

[3] J. Corbett. Constructing abstract models for concurrent real time software. In International Symposium on Software Testing and Analysis, 1996.

[4] P. Cousot and R. Cousot. Abstract interpretation: A unified lattice model for static analysis of programs by construction or approximation of fixpoints. In 4 th ACM POPL, 1977.

[5] D. Dams, O. Grumberg, and R. Gerth. Abstract interpretation of reactive systems: Abstractions preserving $\forall C T L^{*}, \exists C T L^{*}$ and $C T L^{*}$. In E.-R. Olderog, editor, Programming concepts, methods and calculi, volume A-56, pages 573-592. Elsevier, 1994.

[6] R. Duke, G. Rose, and G. Smith. Object-Z: A specification language advocated for the description of standards. Computer Standards and Interfaces, 17:511-533, 1995.

[7] C. Fischer. CSP-OZ: A combination of Object-Z and CSP. In H. Bowman and J. Derrick, editors, Formal Methods for Open Object-Based Distributed Systems (FMOODS '97), volume 2, pages 423-438. Chapman \& Hall, 1997.

[8] C. Fischer and H. Wehrheim. Model-checking CSP-OZ specifications with FDR. In IFM '99: International Workshop on Integrated Formal Methods, Workshops in Computing. Springer, 1999.

[9] Formal Systems (Europe) Ltd. Failures-Divergence Refinement: FDR2 User Manual, Oct 1997.

[10] J.F. Groote and A. Ponse. Proof theory for $\mu$-CRL: A language for processes with data. In Semantics of specification languages, Workshops in Computing. Springer, 1993.

[11] C. A. R. Hoare. Communicating Sequential Processes. Prentice-Hall, 1985.

[12] B. Jonsson and J. Parrow. Deciding bisimulation equivalence for a class of nonfinite state programs. Information and Computation, pages 272-302, 1993.

[13] Kolyang, T. Santen, and B. Wolff. A structure preserving encoding of Z in Isabelle/HOL. In J. von Wright, J. Grundy, and J. Harrison, editors, Theorem Proving in Higher Order Logics, LNCS 1125, pages 283-298. Springer Verlag, 1996. 
[14] C. Loiseaux, S. Graf, J. Sifakis, A. Bouajjani, and S. Bensalem. Property preserving abstractions for the verification of concurrent systems. Formal methods in system design, 6:1-35, 1995.

[15] I. Meisels and M. Saaltink. The Z/EVES Reference Manual. ORA Canada, 1997. http://www.ora.on.ca/z-eves/.

[16] A. Mota and A. Sampaio. Model-checking CSP-Z. In Proceedings of the European Joint Conference on Theory and Practice of Software, volume 1382 of LNCS, pages 205-220, 1998.

[17] J. Quemada, editor. Revised working draft on enhancements to LOTOS (V4). 1996.

[18] A. W. Roscoe. The Theory and Practice of Concurrency. Prentice-Hall, 1997.

[19] A. W. Roscoe, J. C. P. Woodcock, and L. Wulf. Non-interference through determinism. In D. Gollmann, editor, ESORICS 94, volume 875 of LNCS, pages 33-54. Springer-Verlag, 1994.

[20] G. Smith. A semantic integration of Object-Z and CSP for the specification of concurrent systems. In J. Fitzgerald, C. B. Jones, and P. Lucas, editors, Proceedings of FME 1997, volume 1313 of LNCS, pages 62-81. Springer, 1997.

[21] J. M. Spivey. The $Z$ Notation: A Reference Manual. Prentice-Hall International Series in Computer Science, 2nd edition, 1992.

[22] K. Taguchi and K. Araki. Specifying concurrent systems by Z + CCS. In International Symposium on Future Software Technology (ISFST), pages 101-108, 1997.

[23] P. Wolper. Expressing interesting properties of programs in propositional temporal logic. In $A C M P O P L$, pages 184-193, 1986.

[24] J. Woodcock and J. Davies. Using Z. Prentice-Hall International, 1996.

\section{A Brief Introduction to CSP Operators}

We briefly describe the main CSP operators in the syntax that FDR uses.

- SKIP, STOP: empty processes with successful/unsuccessful termination,

- a $\rightarrow$ P: action prefix, first a and then $P$,

$-\mathrm{c} ? \mathrm{x} \rightarrow \mathrm{P}$ : complex prefix, first communication on $\mathrm{c}$ with input value $v$ bound to $\mathrm{x}$, then $\mathrm{P}[v / x]$,

- P; Q: sequential composition,

- P\a: hiding,

- $\mathrm{P}\left[\left[\mathrm{c}<-\mathrm{c}^{\prime}\right]\right]$ : renaming, c "becomes" c',

- P[]Q: external choice,

$-\mathrm{P}|\sim| \mathrm{Q}$ : internal nondeterministic choice,

- b\&P: boolean guard, execute $\mathrm{P}$ if $\mathrm{b}$ evaluates to true,

- P|||Q: interleaving,

- P [I a I]Q: parallel composition with synchronisation on a,

- P [c <-> C'] Q: linked parallel, parallel composition with synchronisation of c and ' ', hiding both channels.

Furthermore most binary operators have an iterated version of the following form (here for []): [] $\mathrm{x}: \mathrm{a} @ \mathrm{P}(\mathrm{x})$ stands for $\square_{x \in a} P(x)$. 


\section{B Proof of Theorem 1}

The proof proceeds via the operational semantics of CSP, i.e. via the generated transition systems and their traces, failures and divergences. We let $\Sigma$ denote a set of visible actions, $\tau$ a distinguished internal action such that $\tau \notin \Sigma$.

Definition 6. A labelled transition system (LTS) is a tuple $T=\left\langle Q, \rightarrow, q_{\text {in }}\right\rangle$, where $Q$ is a set of states, $\rightarrow \subseteq Q \times \Sigma \times Q$ a transition relation and $q_{\text {in }} \in Q$ an initial state.

We write $q \stackrel{a}{\longrightarrow} q^{\prime}$ if $\left(q, a, q^{\prime}\right) \in \longrightarrow, q \stackrel{a_{1} \ldots a_{n}}{\longrightarrow} q^{\prime}$ if there are states $q_{0}, q_{1}, \ldots q_{n}$ such that $q=q_{0}, q_{i} \stackrel{a_{i+1}}{\longrightarrow} q_{i+1}$ and $q_{n}=q^{\prime}$, and $q \stackrel{a}{\Longrightarrow} q^{\prime}$ iff $q \stackrel{\tau^{*}}{\longrightarrow} \stackrel{a}{\longrightarrow} q^{\prime}$ and $a \in \Sigma$. A state $q \in Q$ diverges $(q \uparrow)$ if there are states $q_{1}, q_{2}, \ldots$ such that for all $n \in \mathbb{N}, q_{n} \stackrel{\tau}{\longrightarrow} q_{n+1}$ and $q=q_{1}$.

We prove an even stronger result than Theorem 1 replacing trace refinement by simulation and equivalence by bisimulation. Most results in the area of abstract interpretation for model checking are also based on showing (bi-)similarity of abstract and concrete system. In order to take care about divergences and refusals, we need a special form of bisimulation:

Definition 7. Let $T_{1}, T_{2}$ be transition systems. A relation $\rho \subseteq Q_{1} \times Q_{2}$ is a simulation between $T_{1}$ and $T_{2}$ if $\left(q_{01}, q_{02}\right) \in \rho$ and for all $\left(q_{1}, q_{2}\right) \in \rho, a \in \Sigma$ :

- $q_{2} \stackrel{a}{\Rightarrow} q_{2}^{\prime}$ implies $\exists q_{1}^{\prime}: q_{1} \stackrel{a}{\Rightarrow} q_{1}^{\prime}$ and $\left(q_{1}^{\prime}, q_{2}^{\prime}\right) \in \rho$.

The relation $\rho \subseteq Q_{1} \times Q_{2}$ is a divergence-respecting bisimulation between $T_{1}$ and $T_{2}$ if $\left(q_{01}, q_{02}\right) \in \rho$ and for all $\left(q_{1}, q_{2}\right) \in \rho, a \in \Sigma \cup\{\tau\}$ :

- $q_{1} \stackrel{a}{\longrightarrow} q_{1}^{\prime}$ implies $\exists q_{2}^{\prime}: q_{2} \stackrel{a}{\longrightarrow} q_{2}^{\prime}$ and $\left(q_{1}^{\prime}, q_{2}^{\prime}\right) \in \rho$, and vice versa,

$-q_{2} \stackrel{a}{\longrightarrow} q_{2}^{\prime}$ implies $\exists q_{1}^{\prime}: q_{1} \stackrel{a}{\longrightarrow} q_{1}^{\prime}$ and $\left(q_{1}^{\prime}, q_{2}^{\prime}\right) \in \rho$, and

$-q_{1} \uparrow \Longleftrightarrow q_{2} \uparrow$.

$T_{1}$ can simulate $T_{2}$ (denoted $T_{1} \succeq T_{2}$ ) if there is a simulation from $T_{1}$ to $T_{2}$; $T_{1}$ is d-bisimilar to $T_{2}$ (denoted $T_{1} \approx_{d} T_{2}$ ) if there is divergence-respecting bisimulation between $T_{1}$ and $T_{2}$.

Note the different transition relations used in the definition. A relation $\rho$ is thus not necessarily a bisimulation if both $\rho$ and $\rho^{-1}$ are simulations. The unusual definition of $\stackrel{a}{\Longrightarrow}$ (no further invisible actions after $a$ ) allows for a more convenient proof, we just have to consider the states after one visible step and no further internal moves. This is sufficient for trace refinement.

Proposition 2. Let $T_{1}, T_{2}$ be two transition systems. Then

$$
-T_{1} \succeq T_{2} \Rightarrow T_{1} \sqsubseteq \mathcal{T} T_{2}
$$

$-T_{1} \approx_{d} T_{2} \Rightarrow T_{1}={ }_{\mathcal{F}} T_{2}$. 
Proof of Theorem [1] In the following, we let $Z$ stand for Z_PART and $\|_{\alpha \text { (main) }}$ stand for $[\mid \alpha($ main $) \mid]$. For some given term $Z, h(Z)$ is obtained by replacing in $Z$ all $d$ by $h(d)$ and all enable predicates and effect functions by their abstracted versions. The following general observation is used in all three proofs: for some data-free CSP term $P$, we have: if $P \stackrel{\text { ch.d. } d_{1} \cdot d_{2}}{\longrightarrow} P^{\prime}$ then $P \stackrel{\text { ch.d. } d_{1}^{\prime} \cdot d_{2}^{\prime}}{\longrightarrow} P^{\prime}$ for all $d_{1}^{\prime}, d_{2}^{\prime}$ in the data domain of the channel $c h$. This holds since $P$ only contains communication operations $c h ? x$, thus $P$ can either make any communication over a channel or none.

- The relation proving simulation of $S[G]$ by $S^{\mathcal{S}}$ is

$$
\rho=\left\{\left(Z(h(d)) \|_{\alpha(\text { main })} P,\left(Z(d) \|_{\alpha(\text { main })} P\right)[G]\right) \mid \operatorname{main} \rightarrow^{*} P, d \in D\right\} .
$$

Assume that $\left(Z(d) \|_{\alpha(\text { main })} P\right)[G] \stackrel{c h_{i} \cdot w_{1} \cdot w_{2}}{=}\left(Z\left(d^{\prime}\right) \|_{\alpha(\text { main })} P^{\prime}\right)[G]$. Then $Z(d) \|_{\alpha(\text { main })} P \stackrel{c h_{i} \cdot m_{1} \cdot m_{2}}{=}$ for some $m_{1}, m_{2}$ such that $g_{i}\left(m_{1}\right)=w_{1}$ and $g_{i}\left(m_{2}\right)=w_{2}$. Hence enable $_{c h_{i}}(d)=$ true. By definition of safe abstraction, then enable $c_{h_{i}}^{A}(h(d))=$ true. Furthermore, $m_{1} \in \operatorname{In}\left(c h_{i}\right)$ and $\left(m_{2}, d^{\prime}\right) \in$ effect $_{c h_{i}}\left(d, m_{1}\right)$. Again by definition of safe abstraction we get: $g_{i}\left(m_{1}\right) \in \operatorname{In}^{A}\left(c h_{i}\right)$ and $\left(g_{i}\left(m_{2}\right), h\left(d^{\prime}\right)\right) \in$ effect $\left._{c h_{i}}^{A}(h(d)), g_{i}\left(m_{1}\right)\right)$. Hence $Z(h(d))\left\|_{\alpha(\text { main })} P \stackrel{c h_{i} \cdot w_{1} \cdot w_{2}}{=} Z\left(h\left(d^{\prime}\right)\right)\right\|_{\alpha(\text { main })} P^{\prime}$.

- The relation proving divergence-respecting bisimulation between $S^{\mathcal{O}}$ and $S[G]$ is

$$
\rho=\left\{\left(h(Y) \|_{\alpha(\text { main })} P,\left(Y \|_{\alpha(\text { main })} P\right)[G]\right) \mid \text { main } \rightarrow^{*} P, d \in D\right\},
$$

where $Y$ is either of the form $Z(d)$ or $Y=\square_{c h: O p s}$ enable $e_{c h}(d) \& \square_{\text {in:In(ch) }}$ $X(c h, i n)$, and $X(c h, i n)$ may either be $\Pi_{D I V\left(\text { out }^{\prime} d^{\prime}\right): \text { effect }_{c h}(d, \text { in })}$ ch.in.out $\rightarrow$ $Z\left(d^{\prime}\right)$ or $D I V$ or ch.in.out $\rightarrow Z\left(d^{\prime}\right)$ such that $\left(\right.$ out,$\left.d^{\prime}\right) \in \operatorname{effect}_{c h}(d$, in $)$. Some of the internal choices may have already been resolved (in case of an empty effect set, this might also lead to divergence), while others are not yet taken.

The proof is similar to the above proof, with the difference that we now have to consider single steps (therefore we get all the above terms in our bisimulation relation), and that now the abstract interpretation of the enable and effect predicates match exactly the concrete interpretations. A $\tau$ transition can either be 1) taking an internal choice (resolving $\sqcap$ ) or 2) a divergence. For the divergence we have: effect $c h_{i}(d, m)$ is empty if and only if effect $\operatorname{ch}_{i}^{A}\left(h(d), g_{i}(m)\right)$ is empty. Concerning the resolution of choices: assume that the $\tau$ resolves the choice over all possible values in the effect set of some channel $c h_{i}$ in the concrete system. Then $c h_{i}$ is enabled in the current abstract as well as concrete state. Hence the $\tau$ event occurs in both systems and the resulting processes are in $\rho$ again. 